\title{
Discrete Complex Fuzzy Transform based Face Image Recognition Method
}

\author{
Turker Tuncer \\ Digital Forensics Engineering, Technology Faculty, Firat University, Elazig, Turkey \\ Email: turkertuncer@firat.edu.tr \\ Sengul Dogan \\ Digital Forensics Engineering, Technology Faculty, Firat University, Elazig, Turkey \\ Email: sdogan@firat.edu.tr \\ Erhan Akbal \\ Digital Forensics Engineering, Technology Faculty, Firat University, Elazig, Turkey \\ Email: e@firat.edu.tr
}

Received: 08 January 2019; Accepted: 28 January 2019; Published: 08 April 2019

\begin{abstract}
In this paper, a novel discrete complex fuzzy transform (DCFT) and the proposed DCFT based facial image recognition method is presented. The presented DCFT consists of histogram extraction, peak points of histogram calculation and images construction. 3 real and 3 complex images are constructed using DCFT. Also, 3 angular images and 3 vector image are calculated using the real and complex images. To create real and complex images, polynomial and smith fuzzy sets are used in this paper. Briefly, 12 image are constructed using DCFT. In order to demonstrate effect of the proposed DCFT, face images data sets and local binary pattern (LBP) are used to create facial image recognition method. In this method, LBP is applied on the each DCFT image and $12 \times 256$ size of feature are extracted. Also, maximum pooling is applied on this feature set to obtain 256 size of feature. In the classification phase, support vector machine (SVM) and $\mathrm{k}$ nearest neighborhood (KNN) classifiers are used. The comparisons clearly demonstrate that the proposed DCFT is increased facial image recognition capability.
\end{abstract}

Index Terms-Face recognition, fuzzy transform, classification, local pattern.

\section{INTRODUCTION}

An image consists of pixels and these are divided into two sub-class which are edge and textural [1]. In order to achieve high pattern recognition capability, many edge detectors have been presented in the literature and face recognition and facial image classification are very important research areas for image processing and pattern recognition [2] [3]. In the literature, many texture analysis and recognition methods have been presented and most of them have been used local patterns to feature extraction [4-7]. Also, image transformations such as Wavelet, Fourier, Gabor, Walsh, Schroedinger, etc. have been used to extract meaningful features [8-10]. Facial image recognition has been used the medical, military, biometrics, etc. areas [11-13].

The most famous textural feature extractor is LBP and the LBP uses $3 \times 3$ neighborhood matrix and signum function to binary feature extraction [14] [15]. Then, histogram is extracted and this histogram is utilized as feature. LBP is well discriminator for textural images and it has been used for face, palm print, wood, facial expressions, textural image recognition and map segmentation. LBP has short execution time and it is coded simply by researches because its mathematical background is simple. Briefly, LBP is simple and effective method for facial image recognition. Because of the success of the LBP, many LBP like microstructures have been presented in the literature. Some of these are given as below [16].

\section{A. Related Works}

Zhao et al. [17] proposed a robust version of the LBP because LBP has fragile structure against noises. However, robustness parameter was balanced by users it was not calculated automatically. Song et al. [18] presented an adjacent evaluation of LBP for robust and feature invariant feature extraction. Kaya et al. [19] proposed two LBP version for searching different patterns and they tested these LBP versions onto textural image and butterfly datasets. Mehta and Egiazarian [20] presented a rotation invariant version of the LBP using a reference. This method was fast as the LBP and a dictionary was utilized for feature selection. Chen et al. [21] proposed local convex and concave pattern (LCCP) to eliminate defects of LBP. In order to evaluate performance of the LCCP, palm print, face and textural image datasets were used and the obtained experiments were shown that LCCP has higher textural image recognition capability than LBP but feature dimensionality of LCCP higher than LBP. Liu et al. [22] used two mathematical kernels in LBP and they proposed 
an extended version of the LBP. In this method, angular and radial differences were used instead of pixel values to calculate LBP values. The main objective of this method was to improve facial recognition ability of the LBP. Adnan et al. [23] presented a 1D local ternary pattern (LTP) for fall detection from audio signals. LTP is similar to LBP but LTP uses ternary function to extract binary features and 512 dimensions of feature are extracted using LTP. This method consisted of silent zone suppression, feature extraction with 1D LTP and classification using SVM. They used 2 acoustic datasets and experiment of this study were demonstrated that 1D version of the LTP was successful for audio classification. Liu et al. [24] fused LBP and color image feature (CIF) features to achieve higher textural image classification and retrieval capabilities. Wang et al. [25] used LBP to cloud classification for atmospheric research. Yuan et al. [26] mixed distance based LBP and co-occurrence of LBP features to improve recognition ability. They utilized hamming distance to create a distance based LBP. Textural, smoke image and material datasets were used for evaluation. Singh et al. [27] proposed a color image version of the LBP. HSI color space was used to extract features. Also, uniform patterns were used in this paper. Yang and Yang [28] presented an improved version of the LBP for optical character recognition and they used 4 optical character datasets to evaluate their method. Citraro et al. [29] proposed an extended 3D version of the LBP to detect oxygenated and non-oxygenated brain tissues from MR images for newborn babies

In this paper, a novel complex fuzzy computation based fuzzy transform which called as DCFT is presented and DCFT is used with LBP for facial image recognition. The major contributions and organizations of this paper given as Sections 1.1 and 1.2 respectively.

\section{B. Major Contribution}

The major contributions of this paper are given as below.

- $\quad$ By using polynomial and smith fuzzy sets, a novel complex fuzzy space is obtained.

- A novel complex fuzzy transformation is presented to extract different features of images using this fuzzy space.

- Combining and maximum pooling are utilized as feature fusion. These methods are improved recognition ability.

- $\quad$ The presented textural image recognition method has high image recognition capability.

- This method is the first facial image method using complex fuzzy space in the literature up to now.

- This paper demonstrates that variable fuzzy sets can be used to create complex fuzzy space.

- The proposed method has low computational complexity and it has high classification for facial image classification. These results clearly proved success of this paper.

- A novel lightweight face classification method is presented.

\section{Organization}

The structure of the rest of this paper is given as follows. In the second section Discrete Complex Fuzzy Transform is presented. The proposed facial image recognition method is explained in the Section 3. The experimental results are given in the Section 4 and finally conclusions and recommendations are given in the Section 5.

\section{LOCAL BINARY PATTERN}

LBP is the first known microstructure for textural feature extraction and it has been used various applications of pattern recognition, computer vision and image processing. LBP uses $3 \times 3$ size of neighborhood matrix to extract local features and it uses signum function for feature coding. It has a simple structure. An example about LBP is shown in Fig. 1 [14 [15].

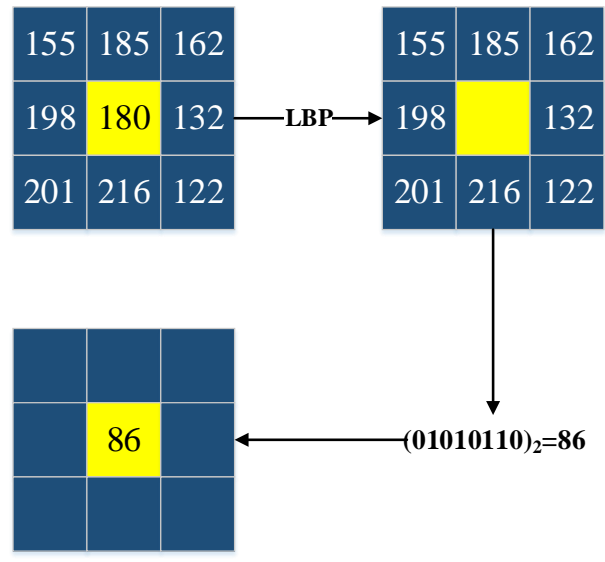

Fig.1. An example about LBP.

In the LBP, firstly image is divided into $3 \times 3$ size of overlapping blocks and center pixel of each block utilized as reference value. By using this reference value and signum function, binary feature coding is applied. Then, histogram of this image is considered as feature vector. To better understanding LBP, description of it given as below mathematically [8] [30].

$$
\begin{gathered}
b_{1}=S\left(p_{i+1, j+1}, p_{i, j}\right) \\
b_{2}=S\left(p_{i+1, j+1}, p_{i, j+1}\right) \\
b_{3}=S\left(p_{i+1, j+1}, p_{i, j+2}\right) \\
b_{4}=S\left(p_{i+1, j+1}, p_{i+1, j}\right) \\
b_{5}=S\left(p_{i+1, j+1}, p_{i+1, j+2}\right) \\
b_{6}=S\left(p_{i+1, j+1}, p_{i+2, j}\right) \\
b_{7}=S\left(p_{i+1, j+1}, p_{i+2, j+1}\right) \\
b_{8}=S\left(p_{i+1, j+1}, p_{i+2, j+2}\right)
\end{gathered}
$$




$$
\begin{aligned}
S(x, y) & =\left\{\begin{array}{l}
0, x-y<0 \\
1, x-y \geq 0
\end{array}\right. \\
L B P^{\text {value }} & =\sum_{k=1}^{8} b_{k} \times 2^{8-k}
\end{aligned}
$$

where $p$ is pixel value, $b$ is bit value, $S($.$) defines signum$ function and $L B P^{\text {value }}$ represents LBP value of the $3 \times 3$ size of block [8] [15].

\section{THE PROPOSED TRANSFORM: DisCRETE COMPLEX FUZZY TRANSFORM (DCFT)}

In this study, novel fuzzy transform which is called as DCFT is presented to extract different and salient features. The steps of the proposed DCFT are given as below.

Step 1: Load image.

Step 2: Extract histogram of the image $H$ with length of 256 .

Step 3: Divide histogram into 3 pieces which are $H_{1}$, $\mathrm{H}_{2}$ and $\mathrm{H}_{3}$ with length of 85,85 and 86 respectively.

Step 4: Calculate indices of the peak points of the $\mathrm{H}_{1}$, $\mathrm{H}_{2}$ and $\mathrm{H}_{3}$ using Eq. 11 .

$$
\left[m_{i} p_{i}\right]=\max \left(H_{i}\right), i=\{1,2,3\}
$$

Where $m_{i}$ are maximum values of each pieces of the histogram and $p_{i}$ are indices of $m_{i}$.

Step 5: Calculate threshold points using the peak points.

$$
\begin{gathered}
t_{1}=p_{1} \\
t_{2}=p_{2}+85 \\
t_{3}=p_{3}+170
\end{gathered}
$$

Where $t_{1}, t_{2}$ and $t_{3}$ are threshold points of the fuzzy sets. 15.

Step 6: Normalize pixel values of the image using Eq.

$$
v_{i, j}=\frac{p_{i, j}}{255}, i=\{1,2, \ldots M\}, j=\{1,2, \ldots, N\}
$$

Where $p_{i, j}$ is pixel values, $v_{i, j}$ is normalized pixel values, $M$ and $N$ represent width and height of the image.

Step 7: Create real and complex fuzzy set using threshold points. Polynomial fuzzy sets represent real and smith fuzzy sets are complex.

$$
\begin{aligned}
& R\left(v_{i, j}\right)=1-\left(\frac{v}{t_{i}}-1\right)^{2} \\
& C\left(v_{i, j}\right)=\frac{1}{1+\frac{\left|v-t_{i}\right|^{2}}{0.15}}
\end{aligned}
$$

Where $R$ and $C$ represent real and complex fuzzy functions.
Step 8: Normalize real and complex values using normalization equation.

$$
v_{i, j}^{N}=\frac{v_{i, j}-v_{\min }}{v_{\max }-v_{\min }}
$$

Where $v_{i, j}^{N}$ normalized value, $v_{\max }$ and $v_{\min }$ represent minimum and maximum value.

Step 9: Apply $R$ and $C$ function to pixel values and calculate real and complex values.

$$
R+C i
$$

3 real and complex images by using Step 9 .

Step 10: Calculate 3 angular and 3 phase images using Eq. 20 and 21.

$$
\begin{gathered}
A_{l}=\arctan \left(\frac{C_{l}}{R_{l}}\right), l=\{1,2,3\} \\
P_{l}=\sqrt{R_{l}^{2}+C_{l}^{2}}, l=\{1,2,3\}
\end{gathered}
$$

The steps 1-10 are clearly explained the proposed DCFT. In the Fig.2, an example about the complex and real fuzzy sets used in this work are shown.

LBP is the first known microstructure for textural feature extraction. LBP has been used various applications of pattern recognition, computer vision and image processing. LBP uses $3 \times 3$ size of neighborhood matrix to extract local features and it uses signum function for feature coding. It has a simple structure. An example about LBP is shown in Fig. 1 [14] [15].

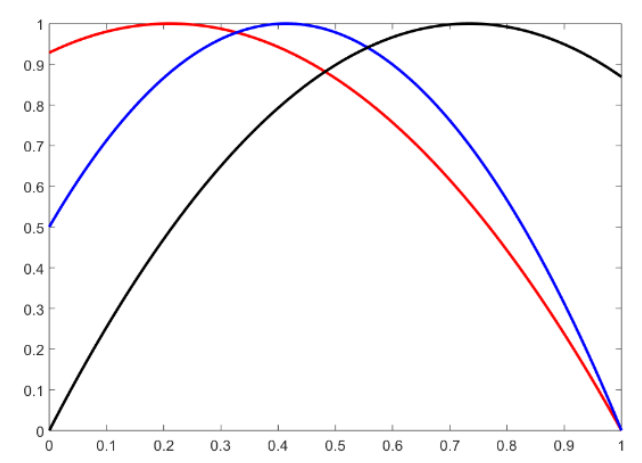

(a)

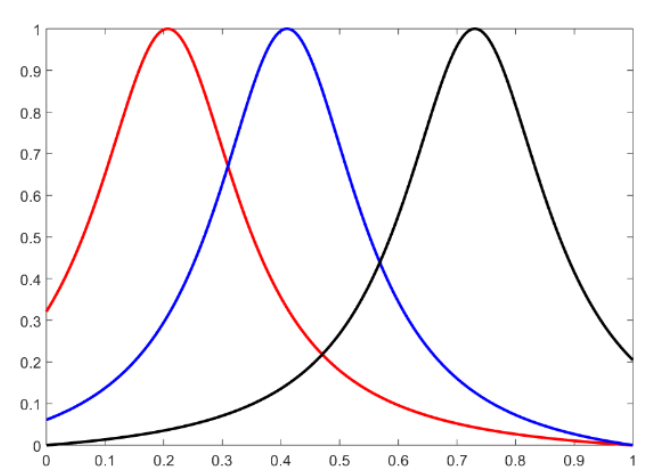

(b) 


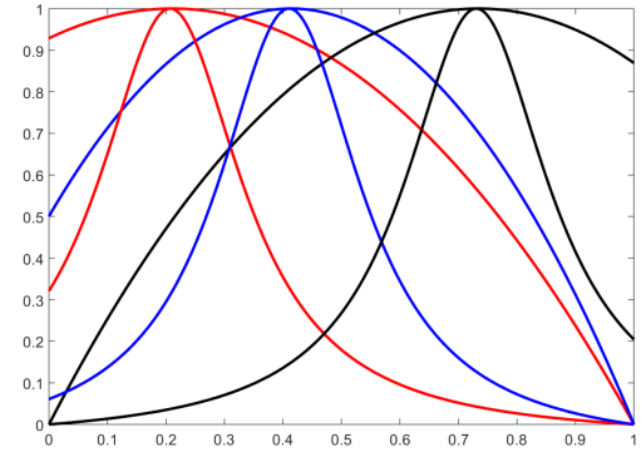

(c)

Fig.2. A The fuzzy sets used in this study (a) real fuzzy sets (b) complex fuzzy sets (c) real and complex fuzzy sets.

12 images are obtained when DCFT was applied on images. These are 3 real images, 3 complex (imaginary) images, 3 angular images and 3 phase images. In the Fig. 3 , an example about the proposed DCFT is shown.

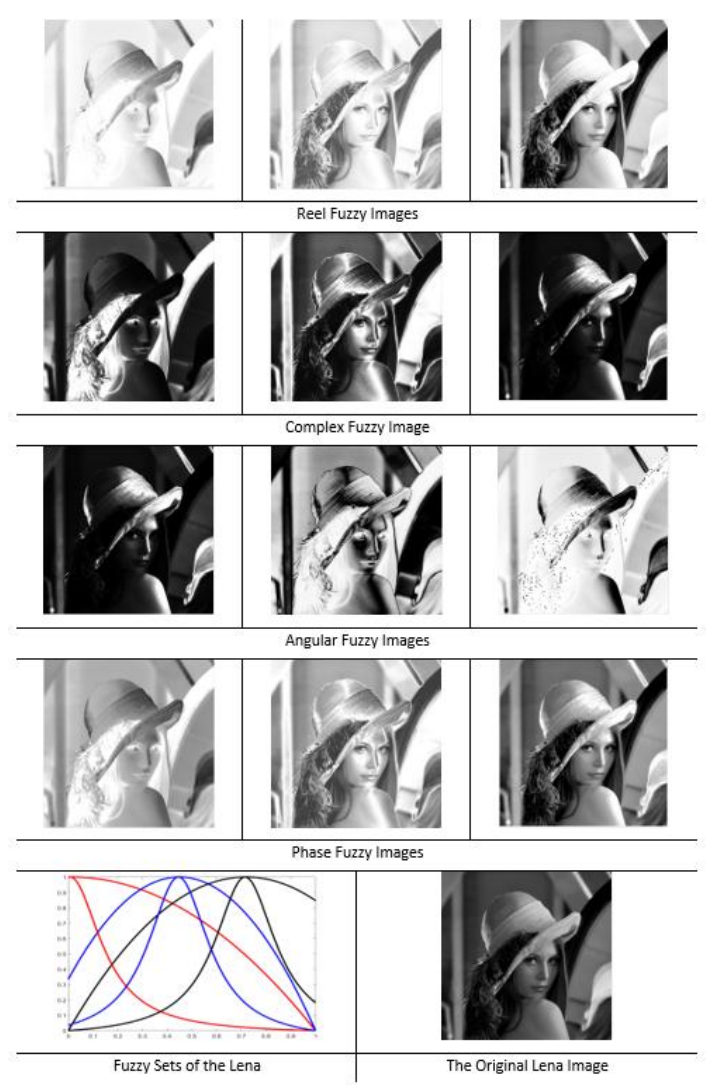

Fig.3. An example about DCFT using Lena.

\section{THE PROPOSED DCFT AND LBP BASED FACIAL IMAGE RECOGNITION METHOD}

In this paper a novel DCFT and LBP based facial image recognition method is presented. In this method, firstly DCFT applied on a cover image then 12 fuzzy images are obtained. To texture feature extraction, LBP is applied on each fuzzy image and histograms of these images are utilized as feature vector. In order to feature fusion, combining and maximum pooling methods are considered. In the classification phase, KNN and quadratic kernel SVM classifiers are used. The graphical outline of the proposed DCFT based method is shown in as Fig. 4.

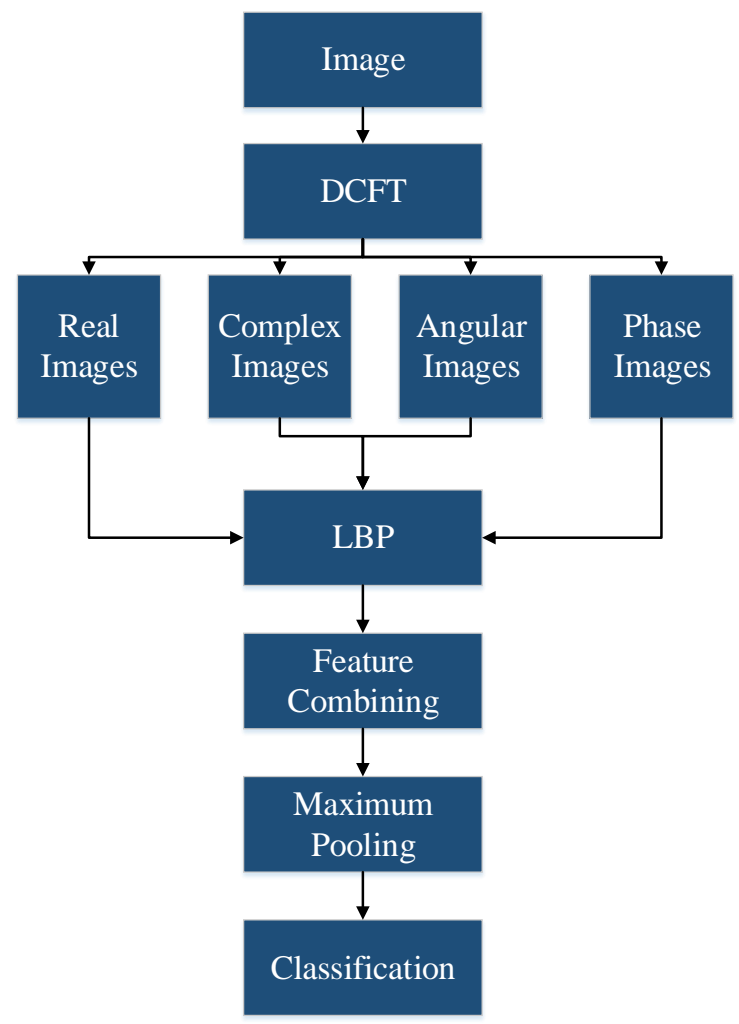

Fig.4. The graphical representation of the proposed DCFT and LBP based texture recognition method.

The steps of the proposed method are given as below.

Step 1: Load image.

Step 2: Apply DFCT and obtain 12 fuzzy images.

Step 3: Calculate LBP of each fuzzy image an obtain 12 feature vector with length of 256 .

Step 4: Combine each vector and obtain $F$ with length of 3072 .

Step 5: Combine histogram real, complex, angular and phase images and obtain $F R, F C, F A$ and $F P$ with length of 768 respectively.

Step 6: Reduce length of $F, F R, F C, F A$ and $F P$ using maximum pooling and obtain $P F, P F R, P F C, P F A$ and $P F P$ with length of 256 . Pseudocode of the maximum pooling on the histogram is lied in Algorithm 1.

Algorithm 1. Pseudo code of the maximum histogram pooling.

Input: Feature vector F with length of $L$

Output: Pooled feature vector PF with length of $L / b$

1: count $=1$;

2: for $\mathrm{i}=1$ to $\mathrm{L}$ step by $\mathrm{b}$ do

3: $\quad$ window $=F(i: i+b-1) ; / /$ Divide feature vector into $b$ length of windows.

4: $\quad P F($ count $)=\max (F)$;

5: $\quad$ count $=$ count +1 ;

6: endfor i 
Step 8: Classify 10 feature vectors obtained using KNN and SVM.

\section{EXPERIMENTAL RESULTS}

The proposed method was programmed by MATLAB 2018a. The extracted features were classified using classification learner toolbox in the MATLAB. KNN and SVM were chosen as classifiers. In this section, 5 face datasets were used to evaluate performance of the proposed method and the widely used methods in the literature were utilized to obtain results. Also, the generated 10 feature vectors which are $F, F R, F C, F A$, $F P, P F, P F R, P F C, P F A$ and $P F P$ were evaluated respectively. In the classification phase, $\mathrm{KNN}$ and quadratic kernel SVM were considered to calculate recognition capability ATT [31], CIE [32], FACE94 [33], FERET [34], MUCT [35], AR [36] face image databases were considered and properties of them listed in Table 1.

Table 1. The properties of the facial databases for performance evaluation.

\begin{tabular}{|c|c|c|c|c|c|}
\hline Database & Classes & $\begin{array}{l}\text { Samples } \\
\text { per Class }\end{array}$ & $\begin{array}{c}\text { Total } \\
\text { Samples }\end{array}$ & $\begin{array}{c}\text { Sample } \\
\text { Resolution }\end{array}$ & $\begin{array}{l}\text { Image } \\
\text { Format }\end{array}$ \\
\hline$A T \& T$ & 30 & 10 & 300 & $92 \times 112$ & pgm $\rightarrow$ jpg \\
\hline CIE & 30 & 10 & 300 & $2048 \times 1536$ & jpg \\
\hline Face94 & 30 & 10 & 300 & $170 \times 512$ & jpg \\
\hline$A R$ & 30 & 10 & 300 & $768 \times 576$ & $\mathrm{raw} \rightarrow \mathrm{jpg}$ \\
\hline$M U C T$ & 43 & 7 & 301 & $480 \times 640$ & jpg \\
\hline FERET & 50 & 6 & 300 & $512 \times 768$ & $\mathrm{ppm} \rightarrow \mathrm{jpg}$ \\
\hline
\end{tabular}

The attributes of classification were given Table 2 .

Table 2. The attributes of classification used in the experimental results.

\begin{tabular}{ccc}
\hline Method & Attributes & Value \\
\hline \multirow{4}{*}{ SVM } & Kernel function & Quadratic \\
& Box constraint level & 1 \\
& Kernel scale mode & Auto \\
& Manuel kernel scale & 1 \\
& Multiclass method & One-vs-All \\
& Standardize data & True \\
& PCA & Disable \\
& Number of neighbors & 1 \\
& Distance metric & City Block \\
& Distance weight & Equal \\
& Standardize data & True \\
& PCA & Disable
\end{tabular}

To obtain image recognition ability measurements, accuracy was utilized as the performance metric and the mathematical description of it is given Eq. 22 [37].

$$
\text { Acc }=\frac{\# \text { Number of true predicted samples }}{\# \text { Number of total samples }}
$$

Where Acc represents accuracy.

The experimental results are given LBP and DCFTLBP in Table 3 and Table 4, respectively.
Table 3. The experimental results for LBP.

\begin{tabular}{ccccccc}
\hline & ATT & CIE & FACE94 & AR & MUCT & FERET \\
\hline SVM & 83.3 & 95.0 & 96.7 & 81.3 & 52.8 & 92.3 \\
KNN & 77.3 & 95.7 & 94.0 & 76.8 & 23.6 & 93.3 \\
\hline
\end{tabular}

Table 4. The experimental results for DCFT-LBP.

\begin{tabular}{ccccccc}
\hline & & PF & PFA & PFC & PFP & PFR \\
\hline \multirow{2}{*}{ ATT } & SVM & 93.3 & 74.3 & 76.0 & 60.3 & 88.0 \\
& KNN & 93.0 & 77.3 & 83.3 & 66.0 & 88.3 \\
\multirow{2}{*}{ CIE } & SVM & 99.0 & 59.0 & 70.3 & 50.7 & 88.3 \\
& KNN & 99.3 & 55.0 & 70.7 & 46.0 & 90.0 \\
FACE9 & SVM & 98.7 & 77.0 & 76.0 & 64.7 & 80.0 \\
4 & KNN & 97.7 & 77.7 & 84.3 & 71.0 & 87.3 \\
& SVM & 92.6 & 76.1 & 75.2 & 65.8 & 83.2 \\
AR & KNN & 86.8 & 78.1 & 82.9 & 69.7 & 88.7 \\
& SVM & 81.8 & 72.8 & 80.4 & 64.8 & 78.4 \\
MUCT & KNN & 75.5 & 74.1 & 78.1 & 66.8 & 84.4 \\
& SVM & 95.0 & 70.3 & 78.3 & 62.3 & 88.7 \\
FERET & KNN & 93.7 & 73.7 & 83.7 & 64.7 & 91.7 \\
\hline
\end{tabular}

Comparison results are given for SVM and KNN classifications in Fig. 5.

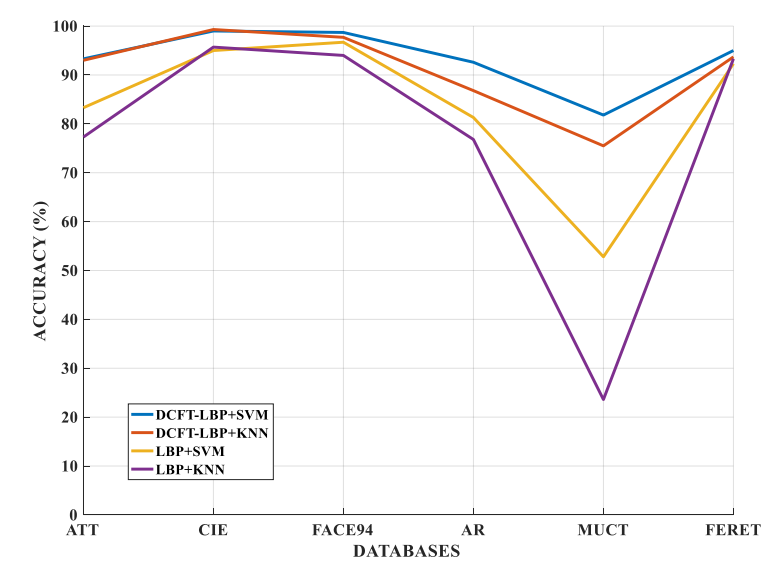

Fig.5. The comparison results of LBP and DCFT-LBP for SVM and KNN.

According to experimental results,

- $\quad$ The proposed DCFT improved the performance of the LBP at high rate.

- $256 \times 12$ dimension of the feature vector was obtained by using DCFT-LBP. Maximum pooling was used to reduce 256 dimension of feature vector. Thus, fair comparison was provided. 5 feature vector which are PF, PFA, PFC, PFP and PFR are obtained using the maximum pooling.

- PF achieved the best performance compared to other vectors (PFA, PFC, PFP, PFR). Therefore, $\mathrm{PF}$ is used in face recognition.

- DCFT can also be used other descriptors.

- The proposed method is an LBP like method. Therefore, LBP was used for comparison. The comparative results are Table 5. 
Table 5. Comparison results of the proposed DCFT-LBP and LBP.

\begin{tabular}{cccc}
\hline Database & Classifier & LBP & $\begin{array}{c}\text { The proposed DCFT- } \\
\text { LBP (PF) }\end{array}$ \\
\hline \multirow{2}{*}{ ATT } & SVM & 83.3 & 93.3 \\
& KNN & 77.3 & 93.0 \\
CIE & SVM & 95.0 & 99.0 \\
& KNN & 95.7 & 99.3 \\
\multirow{2}{*}{ FACE94 } & SVM & 96.7 & 98.7 \\
& KNN & 94.0 & 97.7 \\
AR & SVM & 81.3 & 92.6 \\
& KNN & 76.8 & 86.8 \\
MUCT & SVM & 52.8 & 81.8 \\
& KNN & 23.6 & 75.5 \\
FERET & SVM & 92.3 & 95.0 \\
& KNN & 93.3 & 93.7 \\
\hline
\end{tabular}

PF was used in the comparisons because PF and LBP have 256 sized features. According to Table 5, the proposed method increased success of the LBP for facial image recognition.

\section{CONCLUSIONS AND RECOMMENDATIONS}

In this paper, a novel discrete fuzzy transformation which is called as DCFT and a facial image recognition method to illustrate effect of the DCFT have been presented. The proposed DCFT is the first complex fuzzy transform for the images up to now. The main objective of this transformation is to search different features of the images and increasing recognition ability. By using DCFT, 12 image have been obtained from an image. In the DCFT and LBP based facial image recognition method, DCFT and LBP have been utilized as feature extraction, combining and maximum pooling have been considered as feature fusion and reduction, $\mathrm{KNN}$ and quadratic kernel SVM have been used as classifiers. To obtain numerical results, 5 facial image datasets were used and these experimental results showed that the DCFT increased performance of the LBP.

In future studies, novel image processing methods such as image segmentation, noise removal, pattern recognition, image compression, biometrics applications, etc. may be proposed in the literature and variable fuzzy sets may be used for complex fuzzy space to create novel versions of the DCFT.

\section{REFERENCES}

[1] Turk, M.A. and Pentland, A.P., 'Face Recognition Using Eigenfaces', in, Computer Vision and Pattern Recognition, 1991. Proceedings CVPR'91., IEEE Computer Society Conference on, (IEEE, 1991)

[2] Yang, G. and Huang, T.S., 'Human Face Detection in a Complex Background', Pattern recognition, 1994, 27, (1), pp. 53-63.

[3] Zhang, W., Shan, S., Gao, W., Chen, X., and Zhang, H., 'Local Gabor Binary Pattern Histogram Sequence (Lgbphs): A Novel Non-Statistical Model for Face
Representation and Recognition', in, Computer Vision, 2005. ICCV 2005. Tenth IEEE International Conference on, (IEEE, 2005)

[4] Ahonen, T., Hadid, A., and Pietikainen, M., 'Face Description with Local Binary Patterns: Application to Face Recognition', IEEE Transactions on Pattern Analysis \& Machine Intelligence, 2006, (12), pp. $2037-$ 2041.

[5] Zhang, B., Gao, Y., Zhao, S., and Liu, J., 'Local Derivative Pattern Versus Local Binary Pattern: Face Recognition with High-Order Local Pattern Descriptor', IEEE transactions on image processing, 2010, 19, (2), pp. 533-544.

[6] Jain, A.K. and Li, S.Z., Handbook of Face Recognition, (Springer, 2011)

[7] Ahonen, T., Hadid, A., and Pietikäinen, M., 'Face Recognition with Local Binary Patterns', in, European conference on computer vision, (Springer, 2004)

[8] Suruliandi, A., Meena, K., and Rose, R.R., 'Local Binary Pattern and Its Derivatives for Face Recognition', IET computer vision, 2012, 6, (5), pp. 480-488.

[9] Xu, J., Cha, M., Heyman, J.L., Venugopalan, S., Abiantun, R., and Savvides, M., 'Robust Local Binary Pattern Feature Sets for Periocular Biometric Identification', in, Biometrics: Theory Applications and Systems (BTAS), 2010 Fourth IEEE International Conference on, (IEEE, 2010)

[10] Juefei-Xu, F. and Savvides, M., 'Subspace-Based Discrete Transform Encoded Local Binary Patterns Representations for Robust Periocular Matching on Nist's Face Recognition Grand Challenge', IEEE transactions on image processing, 2014, 23, (8), pp. 3490-3505.

[11] Hassaballah, M. and Aly, S., 'Face Recognition: Challenges, Achievements and Future Directions', IET computer vision, 2015, 9, (4), pp. 614-626.

[12] Kim, T.-K. and Kittler, J., 'Locally Linear Discriminant Analysis for Multimodally Distributed Classes for Face Recognition with a Single Model Image', IEEE transactions on pattern analysis and machine intelligence, 2005, 27, (3), pp. 318-327.

[13] Woodward Jr, J.D., Horn, C., Gatune, J., and Thomas, A., Biometrics: A Look at Facial Recognition', (RAND CORP SANTA MONICA CA, 2003)

[14] Ojala, T., Pietikainen, M., and Maenpaa, T., 'Multiresolution Gray-Scale and Rotation Invariant Texture Classification with Local Binary Patterns', IEEE transactions on pattern analysis and machine intelligence, 2002, 24, (7), pp. 971-987.

[15] Ojala, T., Pietikäinen, M., and Harwood, D., 'A Comparative Study of Texture Measures with Classification Based on Featured Distributions', Pattern recognition, 1996, 29, (1), pp. 51-59.

[16] Shan, C., Gong, S., and McOwan, P.W., 'Facial Expression Recognition Based on Local Binary Patterns: A Comprehensive Study', Image and vision Computing, 2009, 27, (6), pp. 803-816.

[17] Zhao, Y., Jia, W., Hu, R.-X., and Min, H., 'Completed Robust Local Binary Pattern for Texture Classification', Neurocomputing, 2013, 106, pp. 68-76.

[18] Song, K., Yan, Y., Zhao, Y., and Liu, C., 'Adjacent Evaluation of Local Binary Pattern for Texture Classification', Journal of Visual Communication and Image Representation, 2015, 33, pp. 323-339.

[19] Kaya, Y., Ertuğrul, Ö.F., and Tekin, R., 'Two Novel Local Binary Pattern Descriptors for Texture Analysis', Applied Soft Computing, 2015, 34, pp. 728-735. 
[20] Mehta, R. and Egiazarian, K., 'Dominant Rotated Local Binary Patterns (Drlbp) for Texture Classification', Pattern Recognition Letters, 2016, 71, pp. 16-22.

[21] Chen, X., Zhou, Z., Zhang, J., Liu, Z., and Huang, Q., 'Local Convex-and-Concave Pattern: An Effective Texture Descriptor', Information Sciences, 2016, 363, pp. 120-139.

[22] Liu, L., Fieguth, P., Zhao, G., Pietikäinen, M., and Hu, D., 'Extended Local Binary Patterns for Face Recognition', Information Sciences, 2016, 358, pp. 56-72.

[23] Adnan, S.M., Irtaza, A., Aziz, S., Ullah, M.O., Javed, A., and Mahmood, M.T., 'Fall Detection through Acoustic Local Ternary Patterns', Applied Acoustics, 2018, 140, pp. 296-300.

[24] Liu, P., Guo, J.-M., Chamnongthai, K., and Prasetyo, H., 'Fusion of Color Histogram and Lbp-Based Features for Texture Image Retrieval and Classification', Information Sciences, 2017, 390, pp. 95-111.

[25] Wang, Y., Shi, C., Wang, C., and Xiao, B., 'GroundBased Cloud Classification by Learning Stable Local Binary Patterns', Atmospheric Research, 2018, 207, pp. 74-89.

[26] Yuan, F., Xia, X., and Shi, J., 'Mixed Co-Occurrence of Local Binary Patterns and Hamming-Distance-Based Local Binary Patterns', Information Sciences, 2018, 460, pp. 202-222.

[27] Singh, C., Walia, E., and Kaur, K.P., 'Color Texture Description with Novel Local Binary Patterns for Effective Image Retrieval', Pattern recognition, 2018, 76, pp. 50-68.

[28] Yang, C.-S. and Yang, Y.-H., 'Improved Local Binary Pattern for Real Scene Optical Character Recognition', Pattern Recognition Letters, 2017, 100, pp. 14-21.

[29] Citraro, L., Mahmoodi, S., Darekar, A., and Vollmer, B., 'Extended Three-Dimensional Rotation Invariant Local Binary Patterns', Image and vision Computing, 2017, 62, pp. 8-18.

[30] Hurney, P., Waldron, P., Morgan, F., Jones, E., and Glavin, M., 'Night-Time Pedestrian Classification with Histograms of Oriented Gradients-Local Binary Patterns Vectors', IET intelligent transport systems, 2014, 9, (1), pp. 75-85.

[31] Samaria, F.S. and Harter, A.C., 'Parameterisation of a Stochastic Model for Human Face Identification', in, Applications of Computer Vision, 1994., Proceedings of the Second IEEE Workshop on, (IEEE, 1994)

[32] Weeks, A.R., Fundamentals of Electronic Image Processing, (SPIE Optical Engineering Press Bellingham, 1996)

[33] Libor Spacek's Facial Image Database, F.D.h.c.e.a.u.m.a.f.h.a.J., 2018)

[34] Phillips, P.J., Wechsler, H., Huang, J., and Rauss, P.J., 'The Feret Database and Evaluation Procedure for FaceRecognition Algorithms', Image and vision Computing, 1998, 16, (5), pp. 295-306.
[35] Milborrow, S., Morkel, J., and Nicolls, F., 'The Muct Landmarked Face Database', Pattern Recognition Association of South Africa, 2010, 201, (0).

[36] Martinez, A. M. (1998). The AR face database. CVC Technical Report24.

[37] Taheri, S., and Toygar, Ö. 'Animal classification using facial images with score-level fusion. IET Computer Vision, 2018, 12 (5), pp. 679-685.

\section{Authors' Profiles}

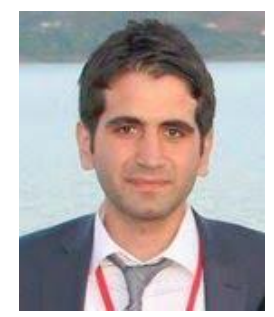

Turker TUNCER was born in Elazig, Turkey, in 1986. He received the B.S. degree from the Firat University, Technical Education Faculty, Department of Electronics and Computer Education in 2009, M.S. degree in telecommunication science from the Firat University in 2011 and Ph.D. degree department of software engineering at Firat University in 2016. He works as research assistant Digital Forensic Engineering, Firat University. His research interests include data hiding, image authentication, cryptanalysis, cryptography, image processing.

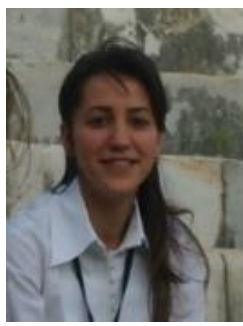

Sengul DOGAN received her Ph.D degree in Electrical and Electronic Engineering from the University of Firat, Elazig, Turkey, in 2011. She is currently an Assistant Professor in the Digital Forensics Engineering Department of Firat University. Her research interests cover Data Hiding, Information Security, Digital Forensics, Image Processing and Optimization Techniques.

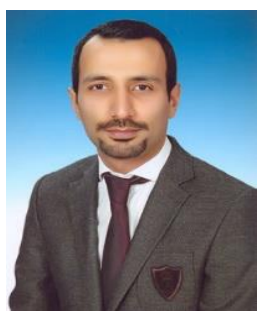

Erhan AKBAL is currently working as an assistant professor at Digital Forensics Engineering Department of Firat University, He received his Ph.D. degree in electrical and electronics engineering in 2012, the M.S. degree in computer engineering in 2007, from Firat University, Turkey. His research interests include computer network security, wireless sensor network, intrusion detection and digital forensics.

How to cite this paper: Turker Tuncer, Sengul Dogan, Erhan Akbal, " Discrete Complex Fuzzy Transform based Face Image Recognition Method", International Journal of Image, Graphics and Signal Processing(IJIGSP), Vol.11, No.4, pp. 1-7, 2019.DOI: 10.5815/ijigsp.2019.04.01 